\title{
Desafección política y medios de comunicación en México. Aproximaciones teóricas, tendencias y hallazgos
}

Political disaffection and the media in Mexico. Theoretical approaches, trends and findings

\author{
Juan Luis Meza Medina \\ Universidad Autónoma de Nuevo León \\ Orcid https://orcid.org/oooo-0002-5987-2079 \\ juan.luis_medina@outlook.es
}

Resumen: En los sistemas democráticos es posible encontrar una diversidad de complejidades que van moldeando la cultura política de los ciudadanos. Dentro de esta cultura política se puede encontrar la desafección política, en donde la ciudadanía desconfía en las instituciones y se aleja de los asuntos relacionados con la política. El presente documento tiene como objetivo elaborar una revisión documental del conocimiento disponible en México sobre los estudios empíricos que han abordado la relación entre efectos mediáticos y desafección política. De la revisión elaborada en el contexto mexicano, se concluye que si bien los paradigmas predominantes son el de malestar mediático (media malaise), así como el de movilización política (political mobilization), los cuales tienen premisas relativamente contrarias, estos se complementan entre sí y los efectos tienden a variar en relación con los niveles de exposición que las audiencias tengan de los diferentes medios y sus contenidos.

Palabras clave: Desafección política, consumo de medios, malestar mediático, movilización política, México

\begin{abstract}
In democratic systems it is possible to find a diversity of complexities that shape the political culture of citizens. Within this political culture, political disaffection can be found, where citizens distrust institutions and move away from issues related to politics. The aim of this document is to prepare a documentary review of the information available in Mexico on empirical studies that have analyzed the relationship between media effects and political disaffection. From the review carried out in the Mexican context, it is concluded that although the predominant paradigms are media malaise and political mobilization, which have relatively contrary premises, these complement each other, and the effects tend to vary in relation to the levels of exposure that audiences have of the different media and their contents.
\end{abstract}

Keywords: Political disaffection, media consumption, media malaise, political mobilization, Mexico

Fecha de recepción: 14/12/2021

Fecha de aprobación: 27/12/2021

Cómo citar este artículo: Meza Medina, J. L. (2021). Desafección política y medios de comunicación en México. Aproximaciones teóricas, tendencias y hallazgos. Revista de Comunicación Política, 3, e210304. https://doi.org/10.29105/rcp3-4 


\section{Introducción}

Dentro de la comunicación política el estudio de los efectos que tienen los contenidos mediáticos en la audiencia que los consume es una línea de gran importancia (Muñiz et al., 2016). Dentro de este campo de estudio sobre los efectos mediático en la ciudadanía destacan dos grandes corrientes teóricas: malestar mediático (media malaise) y movilización política (political mobilization). La primera de ellas con efectos negativos en las audiencias. La segunda sobre efectos positivos.

En este documento se describen y analizan las investigaciones que parten de las corrientes teóricas mencionadas y buscan comprobar sus diversas hipótesis en el contexto mexicano en los últimos diez años. El orden de desarrollo del presente documento inicia con una aproximación teórica al concepto de desafección política y sus características, así como de su importancia dentro del análisis de los sistemas democráticos. Después, se hace un abordaje a las diversas teorías sobre los efectos de los medios en la ciudadanía para dar paso a los trabajos de corte empírico que han estudiado y analizado la relación entre efecto mediático y desafección política en el contexto mexicano.

Por último, es expresa la intención de este trabajo en contribuir a seguir descifrando la compleja trama entre la gran interrogante sobre si los medios de comunicación contribuyen en la generación de desafección política como hace ya más de una década lo planteaba García Luengo (2005) recuperando a Cliff Zukin (1981).

\section{Desafección política en los sistemas democráticos}

Planteaba Pierre Rosanvallon (2006) que el estudio de la democracia es en sí una historia de un desencanto y, a su vez, la historia de una indeterminación. En este sentido, y al respecto de la constante insatisfacción ciudadana con los procesos democráticos y las democracias en general, del Tronco y Monsiváis-Carrillo (2020) señalan que el análisis elaborado por el Democracy Report 2020 refleja que en el periodo que comprende de 2009 a 2019, una gran cantidad de países han mostrado tendencias más cercanas a la autocratización que a la democratización, es decir, en una gran cantidad de países hay síntomas de que la democracia parece haber decepcionado las expectativas de la ciudadanía.

Por ejemplo, es innegable que el común denominador de las encuestas de opinión pública refleja desconfianza por parte de la ciudadanía hacia instituciones políticas, insatisfacción con la forma en la que opera el sistema político y la percepción de que los partidos políticos no representan las demandas de la gente (del Tronco \& Monsiváis-Carrillo, 2020). 
Sin embargo, a pesar del contexto de deterioro y erosión que hay en las democracias, Muñiz y Maldonado (2011) consideran que cabe la posibilidad de encontrar ciudadanos desafectos pero comprometidos, los cuales se caracterizan por tener dudas sobre el sistema pero que participan en él por los mismos mecanismos que este sistema genera impulsados en parte por la información que obtienen.

Dentro de los sistemas democráticos, principalmente los occidentales, se ha hecho cada vez más evidente un aumento generalizado del descontento entre la ciudadanía y, aunado a esto, desde hace décadas existe un debate respecto a una ciudadanía con altos niveles de alienación y un bajo compromiso en el ámbito político (Delli Carpini, 2000). En este sentido, estas condiciones representan un problema para la democracia por la posibilidad latente de un debilitamiento de los sistemas democráticos como consecuencia de una ciudadanía con las características mencionadas (Dalton, 2004; Pharr \& Putnam, 2000).

Para que exista una democracia sólida es indispensable la existencia de una ciudadanía activa e informada, es por tal motivo que, desde la teoría democrática, se considera fundamental que la ciudadanía cuente con altos niveles de sofisticación política y, a la par, ahondar en el estudio de los procesos a través de los cuales la ciudadanía toma decisiones considerando la información de la cual disponen (Dalton, 2006).

Sin embargo, el involucramiento de la ciudadanía no siempre ocurre de manera regular, de esto deriva que la desconfianza que existe en la ciudadanía trasciende más allá del carácter formal de las democracias como son las instituciones, su funcionamiento y el desempeño de los actores políticos. Tal desconfianza se manifiesta a través de una relación endeble entre la ciudadanía, y también, en relación de estos con las autoridades, principalmente con las que en el papel se encargan de los temas de justicia. Además, también destaca una desunión entre la sociedad en ámbitos de la vida que trascienden de categorías como la familia, vecinos y asociaciones de carácter religioso, al igual que cierta desilusión por los logros derivados de la instauración del sistema democrático(Instituto Nacional lectoral [INE], 2015). Todas estas manifestaciones de desconfianza e insatisfacción generalizadas en la ciudadanía trascienden y forman parte de la cultura política de la sociedad.

En este sentido, se entiende por cultura política al "conjunto de actitudes, normas y creencias, compartidas más o menos ampliamente por los miembros de una determinada unidad social y que tienen como objeto fenómenos políticos” (Sani, 1981). Con relación a la definición anterior, la cultura política es un rasgo distintivo que es transmitido por generaciones, a través de los medios de comunicación, mediante las relaciones interpersonales y del entorno en el que están inmersos los individuos (Berger, 1989). 


\section{Desafección política. Conceptualización e importancia para la democracia}

Dentro de la cultura política existen diversas dimensiones y una de ellas es la desafección política. Como sucede de manera general en las ciencias sociales al momento de conceptualizar, la desafección política no es la excepción, ya que se considera un concepto puesto a debate respecto al proceso de conceptualización, así como al momento de articular elementos para su medición (de Vreese, 2005; de Vreese \& Semetko, 2002).

Sin embargo, en los últimos años, la ciencia política en Estados Unidos y Europa señala la existencia de una "crisis de la democracia representativa", misma que se manifiesta como "desafección", es decir, un sentimiento desilusión y distanciamiento que se genera por parte de la ciudadanía hacia el sistema político y los políticos (Pharr \& Putnam, 2000).

Uno de los textos más influyentes en el temade la desafección política en Iberoamérica es el elaborado por Montero et al. (1998) en donde hacen un análisis acerca de las actitudes más relevantes de la ciudadanía hacia el sistema democrático en España durante un periodo de 20 años a partir del año de 1978 y señalan que entre los síntomas más importantes de una degradación del sistema democrático estarían presentes "el desinterés, la ineficacia, la disconformidad, el cinismo, la desconfianza, el distanciamiento, la separación, el alejamiento, la impotencia, la frustración, el rechazo, la hostilidad y la alienación" (p. 25).

El concepto de desafección política se aborda por primera vez por Agger et al. (1961) y lo define como el nivel descrédito de la gente para con los actores políticos y la política. De acuerdo con Torcal y Montero (2006), el término “desafección política” hace referencia:

al sentimiento subjetivo de impotencia, cinismo y falta de confianza en el proceso político, los políticos y las instituciones democráticas que genera un distanciamiento y alienación respecto a éstos, y una falta de interés en la política y los asuntos públicos, pero sin cuestionar el régimen democrático. (p. 6)

En este sentido, la desafección política se conforma por dos dimensiones que son medibles a través de diversos indicadores: la desafección institucional (que se mide por la confianza en las instituciones representativas y la eficacia en la política externa); y la falta de compromiso político (midiendo la eficacia política interna, el interés político y la importancia de la política en la vida) (Torcal, 2006).

Sin embargo, es pertinente señalar que este concepto se podría considerar multidimensional tal y como señala Téllez (2017) al referirse a las tres dimensiones propuestas por Citrin (1977), las cuales son: confianza, responsabilidad y eficacia; las cuatro dimensiones que señala Chen (1992): a) desconfianza o falta de normas, b) ineficacia política, c) dificultad para percibir la divergencia en las 
alternativas políticas y d) la apatía y por último, las propuestas por García Luengo (2009), quien las agrupa en a) afectivas, b) evaluativas, c) cognitivas y d) de conducta. Al respecto, Torcal (2006) plantea la existencia de una diversa gama de hipótesis diversas empleadas para interpretar el aumento en los grados de cinismo político, desconfianza en las instituciones y distintas actitudes que se integran en lo que se ha nombrado como desafección y propone en tres grupos básicos: explicaciones políticas, explicaciones económicas y explicaciones socioculturales.

Ante este panorama, el fenómeno de la desconfianza y distanciamiento ciudadano de la política se torna como una problemática prioritaria que encara la democracia por un inminente efecto en el debilitamiento de los sistemas democráticos (Muñiz, 2019). Por lo tanto, contar con una ciudadanía con bajos niveles de compromiso y tendiente a la alienación se traduce en un incremento de la desafección política que se determina por la desconfianza en las instituciones, distanciamiento de la política y la sensación de no tener la capacidad de incidir en el régimen democrático (Torcal, 2002).

De acuerdo con Austin y Pinkleton (1995), la desafección política se manifiesta mediante una postura desfavorable respecto a los asuntos políticos , misma que, como señala Muñiz (2019) desencadena un proceso de espiral de desafección que abre la posibilidad a la generación de condiciones adversas a la democracia como lo son el cinismo, alienación, desconfianza y apatía, o, cualidades positivas como el escepticismo, factor importante para incentivar la participación política (Yamamoto \& Kushin, 2014).

De esta serie de cualidades, tanto positivas como negativas, es preciso puntualizar en cuatro: cinismo, apatía, escepticismo y confianza política. Sobre el cinismo político, Paramio (1999) lo define "como una forma de actuar en que se percibe que los políticos no se ocupan de los problemas de la gente común, sino de sus propios intereses" (p. 3). Respecto a la apatía política, Yamamoto y Kushin (2014) consideran que esta se interpreta como la negatividad plena en a todos los asuntos relacionados con los asuntos políticos y, en lo que concierne al escepticismo, este se manifiesta como la falta de credibilidad sobre el sistema en general, sin embargo, esta incredulidad no manifiesta rechazo al mismo. Al respecto de la confianza política, del Tronco (2012) considera que esta es uno de los pilares de la democracia representativa, ya que es dicha confianza el puente entre los ciudadanos y las instituciones, mismas que están diseñadas para representar tanto necesidades como intereses de la ciudadanía en general.

Para el caso mexicano, Maldonado (2013) señala que las características que tiene la desafección política como tal representan un reto para la calidad del régimen democrático del país, ya que acentúan aspectos como la desigualdad social, impiden la participación política informada y restringen que los ciudadanos ejerzan control de instituciones y políticos. 


\section{Medios de comunicación y democracia}

Respecto a los medios de comunicación, se dice que estos son medios que emiten información por la vía de canales diversos como prensa, radio, televisión e internet, existiendo en sus contenidos variedad en la información y en la que se incluye la relacionada a temas sobre política (Saldierna, 2014). Por una parte, los medios tradicionales en los que se incluye la prensa, radio y televisión, y, por otra parte, los medios sociales, los cuales se caracterizan por ser a través de internet: Facebook, Twitter, YouTube, Instagram y WhatsApp.

En este sentido de la importancia de los medios de comunicación como fuente de información de la ciudadanía para la toma de decisiones entorno a los temas de política, un aspecto fundamental es el consumo de medios de comunicación, entendido como el instrumento con el que cuentan los ciudadanos para vincularse con cuestiones públicas (Espinoza-Bianchini, 2018).

A grandes rasgos, los medios de comunicación cuentan con gran relevancia para que el compromiso político de los ciudadanos se haga presente o se mantenga constante en relación con el funcionamiento del sistema político (García Luengo \& Maurer, 2009). Dentro de los medios tradicionales, destaca la televisión como el gran referente del siglo XX en cuanto a medios de comunicación masivos con la capacidad de influir en los individuos.

Por lo tanto, cuando se habla de medios, se hace referencia a los ámbitos de comunicación masiva que difunden mensajes a amplias audiencias a través de la prensa, radio, cine y televisión (Trejo, 2000). Los medios de comunicación masiva comprenden las instituciones y técnicas con las que especialistas utilizan la tecnología con la finalidad de propagar mensajes en el entorno de grandes audiencias de características diversas (Janowitz, 1952, citado en McQuail, 1972). En este sentido, otro gran referente con mayor tiempo de existencia ha sido la prensa escrita, la cual ha estado vigente incluso con todo el avance de los medios digitales al igual que la radio complementándose con la llegada de internet se potencializó el consumo de medios digitales.

El papel que han desempeñado los medios de comunicación en las democracias ha generado una importante línea de investigación. Los estudios y aportes teóricos que existen en el ámbito de los efectos de los medios de comunicación en la ciudadanía y los sistemas democráticos tienen origen en dos grandes paradigmas: el malestar mediático (media malaise) y la movilización política (political mobilization) (García Luengo, 2009).

Es con el incremento de la importancia de la televisión durante los años setenta del siglo XX que los estudios sobre malestar mediático (media malaise) cobran fuerza en los Estados Unidos (Avery, 2009; Cappella \& Jamieson, 1997; de Vreese, 2005). Dichos estudios se caracterizaban por el análisis de los efectos negativos, producto del consumo de medios relacionados con temas políticos, en los ciudadanos (Muñiz \& Maldonado, 2011; Rojas, 2006). 
Por otra parte, es en los años noventa cuando se empiezan a desarrollar estudios enfocados en los posibles efectos del consumo de medios en las actitudes políticas de los ciudadanos, los cuales mostraban una perspectiva optimista en contraste con el malestar mediático (de Vreese, 2005; Rojas, 2006). A este nuevo paradigma se le conoce como movilización política (political mobilization), el cual se caracteriza por su posibilidad de aumentar los niveles de involucramiento político de la ciudadanía (Holtz-Bacha, 1990), en el cual destaca la teoría del círculo virtuoso (Norris, 2000).

\section{Malestar mediático (Media malaise)}

Es después de la mitad del Siglo XX cuando se comenzaron a plantear diversos estudios enfocados principalmente en la idea de que los contenidos mediáticos influían en la generación de efectos negativos en temas relacionados con el aspecto político en las democracias, reflejándose en aspectos como desconfianza, y desinterés en el funcionamiento del sistema político en general teniendo como resultado el paradigma del malestar mediático o videomalestar (media malaise) (Avery, 2009).

Dentro de los estudios del malestar mediático, Saldierna (2014) plantea que las dinámicas dentro de la comunicación política manifiestan un efecto sustancial en el compromiso cívico y que dicha influencia se manifiesta de forma negativa. Además, resalta que estos efectos negativos pueden ser un riesgo latente para la sociedad en general ya que desencadenan en desinterés sobres los asuntos públicos.

Si bien el primer término para hacer referencia al impacto negativo de los medios de comunicación en la ciudadanía fue el de videomalestar (videomalaise) acuñado por Robinson (1976) por la preponderancia que entre los años setenta y ochenta tuvo la televisión, para los propósitos de este documento se hablará de malestar mediático (media malaise) pugnando por la justificación propuesta por García Luengo (2005) al contemplarlo como "un término más completo, menos discriminante, con la intención de contemplar la trascendencia de otros medios como los escritos en este proceso de desencanto político" (p. 11).

\section{Movilización política (political mobilization)}

Si bien el paradigma de malestar mediático cuenta con una influencia notable en el estudio de los efectos mediáticos, principalmente a partir de los años setenta del siglo XX, es durante la década de los noventa que surge el paradigma denominado de movilización política (political mobilization) que contemplaba que los efectos del consumo mediático en las actitudes políticas de la ciudadanía podían ser positivos e incluso incrementar el involucramiento político de los ciudadanos (Avery 2009; Cappella \& Jamieson, 1997; Holtz-Bacha, 1990).

En este paradigma sobresale la teoría del círculo virtuoso planteada por Norris (2000) y establece que los ciudadanos con mayor actividad política son más propensos al consumo de niveles de 
información política mayores, lo que se traducirá en un incremento en su activismo. Es decir, "las personas que ven más informativos de televisión leen más periódicos, usan Internet y prestan atención a las campañas electorales, tienen consistentemente más conocimiento, confianza en el gobierno y son más participativos" (Norris, 2001, p. 25).

En síntesis, las teorías del malestar mediático asumen dos postulados clave para entender su importancia. El primero establece que los procesos de comunicación política influyen de forma significativa en el compromiso cívico de la ciudadanía. El segundo, concluye que el impacto que dichos procesos se manifiesta de forma negativa, es decir, que la escasez de compromiso con el proceso político tiende a determinarse por el proceso de comunicación política (García Luengo, 2005).

\section{Estudios en México}

Para el caso de los estudios realizados en México, en la literatura revisada sobre trabajos empíricos, las investigaciones destacan por plantear de forma explícita el objetivo de poner a prueba las hipótesis de la movilización y el malestar mediático y han encontrado evidencia mixta que las sustenta. Los trabajos realizados han abordado diversas dimensiones de la desafección política como la apatía, el cinismo, el escepticismo y la confianza y dentro de estas investigaciones hay una variedad de características que las enriquecen como su enfoque durante periodos no electorales y electorales, así como en diferentes grupos de la población (preciudadanos, jóvenes y población en general).

$\mathrm{Al}$ respecto de estudios llevados a cabo en periodos no electorales, Corduneanu y Muñiz (2011), mediante una encuesta representativa a nivel nacional realizada vía telefónica, analizaron el impacto del consumo y nivel de exposición a medios en algunos elementos de la legitimidad democrática como confianza política, interés en la política, eficacia política, valoración de la democracia y preferencias respecto de un régimen autoritario. En esta investigación los autores destacan la alta valoración que la población otorgan a instituciones como el Ejército y la Iglesia. Respecto a la valoración del Ejército, esta fue superior en todos los grupos de consumo, sobresale en audiencias de radio y televisión, en contraste con los ciudadanos que leen prensa quienes mostraron niveles menores de confianza frente a esta institución. En este sentido, son los televidentes y los radioescuchas quienes muestran los mayores grados de confianza en las instituciones. Por lo tanto, en este estudio los autores concluyeron que sus hallazgos ponen en entredicho, e incluso contradicen, los postulados de la teoría del malestar mediático al no coincidir con las aseveraciones en las que se plantea a la televisión como el medio responsable de los niveles de cinismo político y de una baja confianza en las instituciones, aunado a la confirmación de la teoría del círculo virtuoso que plantea que entre más interés, más alto es el consumo, lo que retroalimenta el interés en política (pp. 304-306). 
En este sentido, Aruguete y Muñiz (2012) utilizaron datos provenientes de la Encuesta Nacional sobre Cultura Política y Prácticas Ciudadanas (ENCUP), elaborada por la Secretaría de Gobierno de México en el año 2008. El diseño elaborado para la muestra de esta encuesta fue probabilístico, trietápico, estratificado y por conglomerados y se consideró la edad de 18 años cumplidos al momento de aplicación de la encuesta. En este estudio, los autores detectaron que la población mexicana de mayor de edad acude con más regularidad a la televisión que a la prensa para consumir información acerca de política y hallaron evidencia que respalda a la teoría de la movilización política a través de un análisis de regresión demostrando que el consumo mediático contribuyó en forma positiva al aumento de la confianza política destacando que el consumo de noticias a través de la radio tuvo un leve impacto significativo en la confianza en las instituciones políticas (pp. 142-144).

También han sido elaborados estudios con jóvenes universitarios. Tal es el caso de Leyva et al (2016), quienes analizan la participación político-electoral de los jóvenes universitarios de 18 a 30 años en el estado de Nuevo León a través de un muestreo probabilístico. El estudio se centró en tres hipótesis de investigación enfocadas en analizar la influencia de variables como consumo de medios, actitudes políticas e identificación partidaria determina o explican los niveles de participación político-electoral de los jóvenes. Respecto a la desafección política en su manifestación de cinismo, al evaluar las actitudes políticas de los jóvenes el resultado fue un cinismo ligeramente alto $(M=3 \cdot 37$, $D E=1.16)$ que, al tomar en cuenta la tesis del malestar mediático, dicho resultado se podría atribuir al consumo de medios masivos (p. 64). En este sentido, se concluye que el consumo de medios, particularmente a través de la atención a contenidos en medios digitales aumenta el desarrollo de la participación, en contraparte del impacto de los medios tradicionales los cuales no inciden significativamente, y destacando, también, que la confianza resultó ser el factor más importante de las actitudes políticas que explican el desarrollo de la participación, así como el interés y la eficacia (p. 68).

Por otra parte, sobre investigaciones realizadas durante periodos electorales, se encuentra la investigación de Díaz Jiménez y Muñiz (2017) en donde analizan, en el contexto de la elección presidencial en México de 2012, el efecto mediático en el compromiso político de los jóvenes, siendo la confianza política el elemento de la desafección política medido a través de la Encuesta Nacional sobre Cultura Política de los Jóvenes 20012 realizada por el entonces IFE y el Colegio de México que se aplicó a jóvenes de 18 a 29 años de edad. Este análisis multivariado de la Encuesta arrojó tres hallazgos importantes. El primero, sobre los grados mínimos de compromiso político referente a las juventudes de centros urbanos en México, abarcando índices de participación electoral, discusión política, conocimiento político, eficacia política, pertenencia a organizaciones sociales y confianza política.

El segundo hallazgo da muestra de audiencias juveniles que prestan poca atención a información referente a la política y que se enfoca prioritariamente al entretenimiento mediático, lo cual, a pesar del tipo de contenido este no influyó de forma negativa en los patrones de compromiso político de las audiencias juveniles, incluso, se detectó que el entretenimiento a través de la televisión reflejó una asociación de carácter significativa y a su vez positiva con relación a los niveles de participación 
comunitaria en los jóvenes . Y, el tercer hallazgo, respecto a que la información de las campañas que emitieron los partidos políticos a través de los tres tipos de canales de comunicación política utilizados para el estudio (canales de contacto intensivo con la gente, canales basados en medios masivos y canales basados en Internet) se inclinó a reforzar el compromiso cívico de los jóvenes, incluyendo sus niveles de conocimiento político y confianza política (p. 209-210). En conclusión, los autores plantean que los resultados de su investigación tienden a vincularse más con las teorías de la movilización, especialmente, con la teoría del círculo virtuoso.

Por su parte, Marañon et al (2017) buscaban identificar por medio de una investigación cuasiexperimental el tipo de ruta persuasiva en la publicidad política mediante spots con mayor influencia en la desafección política, en donde esta comprendía cinismo, apatía y reflexión. En dicho estudio, donde se utilizaron dos tipos de spots, uno con ruta periférica y otro con ruta central, se constató que en los dos grupos existió una alta tendencia a la desafección, siendo los participantes expuestos a la ruta central los que en mayor medida lo manifestaron y en menos medida aquellos quienes se expusieron al spot de ruta periférica. En este sentido, y como conclusión general, se considera que la desafección política es generada en gran medida debido a la cantidad excesiva de publicidad política sobre un candidato cuando esta incluso no llega a formar parte de los temas de conversación dentro la cotidianeidad del ciudadano. Es por tal motivo que, a pesar de que el objetivo fundamental de la utilización de las rutas central y periférica esté orientado a la persuasión del votante y este se ve envuelto en la desafección política se estima que con mayor facilidad una ruta central con enfoque de ataque repercuta en él, , reforzando el distanciamiento y desafección que siente hacia el gobierno en turno, dando como resultado que la persuasión pueda ser más efectiva y duradera (pp. 155-156).

A su vez, Muñiz (2019) analiza cómo y en qué grado el efecto de los medios en las actitudes políticas trazado a través del modelo O-S-R-O-R propuesto por Shah et al. $(2005,2007)$ se manifestó en las elecciones presidenciales en México del año 2018. En esta investigación se estudia la desafección política en sus manifestaciones de apatía, cinismo y escepticismo con un diseño metodológico explicativo a través del método de encuesta panel donde se analizaron las actitudes de una población y el cambio en estas a lo largo del tiempo, así como las variables que determinan dicha evolución. En primer lugar, el estudio buscaba analizar si las relaciones entre cinismo, apatía y escepticismo identificadas en los ciudadanos estaban más orientadas hacia una espiral de desafección positiva o, tal vez, negativa, concluyendo sobre la presencia del proceso de desafección al encontrarse relación entre el cinismo político con las variables apatía y escepticismo. Sin embargo, no se observó una asociación entre escepticismo y apatía.

En segundo lugar, en el artículo se plantearon dos modelos teóricos para cada variable criterio en relación de si las variables que fungieron como mediadoras, eran online u offline. Estos dos modelos, a través de preguntas e hipótesis de investigación, buscaban contrastar, por una parte, la existencia de un efecto indirecto del interés hacia la política sobre cinismo, apatía y escepticismo, a través de medios tradicionales y, por otra parte, a través de medios sociales. Los resultados principales de esta 
investigación plantean un incremento de la apatía influido por el consumo de medios tradicionales, un cinismo poco afectado por los medios de comunicación y, respecto al escepticismo, los resultados no demostraron ser tan concluyentes como con la apatía y su relación con los medios. En síntesis, el trabajo concluye que el consumo mediático tiene una incidencia favorable en la configuración de audiencias que proponen interrogantes sobre la información que reciben y que indagan información complementaria sobre la ya que han obtenido con la finalidad de completar su conocimiento, formando parte de un proceso de espiral de desafección con resultado más positivo (pp. 101-103)

Dentro de estas investigaciones, también se ha trabajado con sujetos de estudio que en su mayoría aún no han participado en procesos electorales como votantes, lo cual es interesante porque permite conocer la evolución a lo largo del tiempo de diversos aspectos de la cultura política de los jóvenes.

Por ejemplo, Muñiz y Maldonado (2011), en un estudio destacado en este tema, encontraron la existencia de un alto cinismo político, o desafección, hacia el sistema político. Dicha investigación se llevó a cabo mediante una encuesta a una muestra representativa de la población de estudiantes de bachillerato de 15 a 18 años en la Zona Metropolitana de Monterrey. Los autores destacan en los resultados que el cinismo político no fue explicado por la exposición a medios como la televisión y la atención a programas de entretenimiento, sino por la atención a programas sobre política y a la exposición a medios como prensa e internet, sin embargo, estos resultados tienden a reflejar un cinismo comprometido que se vincula más con prácticas participativas de aquellas personas que son más interesadas y se informan más sobre política (p. 51).

De igual manera, es para el caso de otros estudios que también han analizado estudiantes de bachillerato de 15 a 18 años como Saldierna y Muñiz (2014), quienes detectaron evidencia de cinismo político al concluir que los jóvenes consideran que nada pueden hacer en lo que se refiere al sistema político (p. 224), así como Saldierna y Muñiz (2015), quienes a través de una encuesta aplicada a una población de estudiantes que cursaban el último año de bachillerato en la Zona Metropolitana de Monterrey, Nuevo León, México, analizaron la influencia que tienen diversos hábitos comunicativos de los preciudadanos (jóvenes de 15-18 años), como lo son consumo de diversos tipos de medios y conversación política interpersonal e interactiva, en la cultura política de estos, especialmente en aspectos como la participación política y el cinismo político, concluyendo que, en relación a la exposición mediática, la televisión no ejerce ninguna influencia en la generación de cinismo político, al igual que la evidencia del uso casi nulo de la prensa escrita en este grupo de la población (p. 55).

Por último, también destaca una investigación de corte cualitativo en la que Echeverría (2011) explora la apatía política a través de un estudio cualitativo compuesto por grupos focales, para jóvenes de clase media, y entrevistas semi estructuradas, para jóvenes de clase popular en Mérida, Yucatán, en el periodo de diciembre 2008 a febrero 2009. En esta investigación se abordaron cuestionamientos relacionados con los hábitos de consumo de medios presente en los jóvenes y la desconfianza de este 
grupo de la población hacia actores políticos concluyendo acerca de actitud frecuentemente desinformada de los jóvenes se distingue por un cinismo que podría entenderse de forma crítica y de escepticismo (pp. 61-62).

\section{Reflexiones finales}

Como se planteó en este trabajo, los sistemas democráticos, ya sea consolidados o en transición, son inherentes a una serie de complejidades que van configurando la cultura política de sus ciudadanos misma que se manifiesta de diversas maneras como lo puede ser la desafección política. Por ello, resulta pertinente entender a profundidad los procesos mediante los cuales la ciudadanía se relaciona con el sistema político, ya que dicha relación es fundamental para que los sistemas democráticos sigan siendo una alternativa viable para que los ciudadanos ejerzan con plenitud sus derechos.

El presente estudio buscaba elaborar una revisión documental del conocimiento disponible en México acerca de la relación de los efectos mediáticos con la desafección política y las diversas manifestaciones de esta ya que, si bien existe una gran cantidad de trabajos en los que se analizan aspectos como la participación política en sus diversos aspectos, es el concepto de desafección política otro elemento de la cultura política poco explorado en cuanto a su relación con los medios.

En este caso, y de manera general, con la revisión de literatura planteada en el presente documento, se puede observar la diversidad en los estudios realizados en el contexto mexicano en los que se aborda la desafección política en alguna de sus dimensiones, en donde dichos trabajos han abordado en su mayoría la variable de cinismo político. Las investigaciones se han realizado en contextos electorales, no electorales, en diferentes grupos de la población como adolescentes de 15-18 años, jóvenes y adultos, desde lo local a lo nacional. Incluso, de forma longitudinal con muestras representativas a través de una encuesta panel.

Por lo tanto, al respecto de las investigaciones abordadas en este trabajo y elaboradas en el contexto mexicano, el resultado de esta revisión documental permite plantear que, tanto el paradigma de malestar mediático (media malaise), así como el de movilización política (political mobilization) y del círculo virtuoso, tienden a retroalimentarse entre sí, resultando más evidentes las diferencias en los efectos en función de la exposición que las audiencias tengan de los diferentes medios y sus contenidos.

Por ejemplo, Muñiz y Maldonado (2011) señalan la existencia de un cinismo comprometido, más bien orientado al escepticismo y a la búsqueda de información, que a la apatía política. Por su parte, Corduneanu y Muñiz (2011) plantean que si bien con los hallazgos de su estudio confirman los postulados de la teoría del círculo virtuoso de que entre más interés más alto será el consumo también encuentran una tendencia a apoyar un régimen autoritario, hallazgo más cercano a la teoría del ma- 
lestar mediático, el cual se ve influido por los medios tradicionales, en contraste con quienes manifestaron menor apoyo a este tipo de régimen, que se caracterizaban por indicar el Internet como su fuente principal de información. Es decir, y poniendo como ejemplo estas dos investigaciones, sin dejar de lado las cualidades y características de los demás estudios consultados, que se puede concluir que la evidencia empírica comprueba la existencia de los efectos mediáticos en las actitudes políticas de los ciudadanos, en este caso la desafección política, de forma complementaria entre ambos paradigmas, marcando la diferencia el tipo de medio y el contenido al que se exponen los ciudadanos.

Por otra parte, también es preciso señalar, que respecto a las dimensiones más exploradas de la desafección política en los documentos analizados se encuentran la confianza y el cinismo político como variables. En este sentido, sobresale dentro de la comunicación política la ausencia de estudios de carácter cualitativo que permitan ampliar el panorama sobre el entramado que existe entre la presencia de desafección política en la ciudadanía. Sin embargo, es necesario reconocer que el estudio de los efectos de los medios en México se ha venido consolidando con estudios empíricos de enfoque cuantitativo como los que se han descrito para fines de este trabajo. Sin duda, los paradigmas de malestar mediático y movilización política han permitido conocer de forma detallada los procesos de obtención de información de los ciudadanos y cómo se manifiesta en sus actitudes y comportamientos. Por lo tanto, es necesario seguir explorando la relación entre desafección política y medios de comunicación desde diversos enfoques metodológicos para poder conocer a profundidad este aspecto de la cultura política.

En síntesis, el propósito del presente trabajo radica en ahondar en el vínculo existente entre la desafección política y su relación con los medios de comunicación vista desde el campo de la comunicación política y así poder contribuir de forma propositiva al debate sobre los aspectos teóricos y empíricos de esta línea de investigación.

\section{Declaración de conflicto de intereses}

El autor no informó ningún posible conflicto de intereses.

\section{Fondos}

Este artículo científico deriva de la tesis de doctorado titulada "Las prácticas comunicativas como factores determinantes de la desafección politica en jóvenes y su efecto en el involucramiento político", investigación apoyada a través de una Beca CONACYT. Asimismo, fue desarrollado en el marco del proyecto de investigación titulado "Análisis de la cobertura mediática de las campañas electorales

y su impacto en la desafección política y el compromiso democrático ciudadano” (Clave 280739), aprobado por CONACYT dentro de la Convocatoria de Investigación Científica Básica 2016. 


\section{Referencias}

Agger, R. E., Goldstein, M. N., \& Pearl, S. A. (1961). Political cynicism: Measurement and meaning. The Journal of Politics, 23(3), 477-506. http://doi.org/10.2307/2127102

Aruguete, N., \& Muñiz, C. (2012). Hábitos comunicativos y política. Efectos en las actitudes políticas de la población mexicana. Anagramas. Rumbos y Sentidos de la Comunicación, 10(20), 129-146. http://dx.doi.org/10.22395/angr.v10n20a9

Austin, E. W., \& Pinkleton, B. E. (1995). Positive and negative effects of political disaffection on the less experienced voter. Journal of Broadcasting \& Electronic Media, 39(2), 215-235. https://doi.org/10.1080/08838159509364300

Avery, J. M. (2009). Videomalaise or virtuous circle? The influence of the news media on political trust. The International Journal of Press/Politics, 14(4), 410-433. http://doi.org/10.1177/1940161209336224

Berger, A. (1989). Political culture and public opinion. Transaction Publishers.

Cappella, J. N., \& Jamieson, K. H. (1997). Spiral of cynicism: the press and the public good. Oxford University Press.

Chen, K. (1992). Political alieanation and voting turnout in the United States 1960-1988. Mellen Research University Press.

Citrin, J. (1977). Political alienation as a social indicator: Attitudes and action. Social Indicators Research, 4(1), 381-419. https://doi.org/10.1007/BFo0353142

Corduneanu, V. I., \& Muñiz, C. (2011). ¿Autoritarismo superado? Medios y actitudes políticas en el contexto mexicano. En C. Muñiz (Ed.), Comunicación, política y ciudadanía. Aportaciones actuales al estudio de la comunicación política (pp. 283-307). Fontamara.

Dalton, R. J. (2004). Democratic challenges, democratic choices: The erosion of political support in advanced industrial democracies. Oxford University Press. http://doi.org/10.1093/acprof:oso/9780199268436.001.0001

Dalton, R. J. (2006). Citizen politics: public opinion and political parties in advanced industrial democracies ( $4^{\mathrm{a}}$ ed.). CQ Press.

de Vreese, C. H. (2005). The spiral of cynicism reconsidered. European Journal of Communication, 2O(3), 283-301. http://doi.org/10.1177/0267323105055259 
de Vreese, C. H., \& Semetko, H. A. (2002). Cynical and engaged: Strategic campaign coverage, public opinion, and mobilization in a referendum. Communication Research, 29(6), 615-641. http://doi.org/10.1177/009365002237829

del Tronco, J. (2012). Las causas de la desconfianza política en México. Perfiles Latinoamericanos, $20,227-251$.

del Tronco, J., \& Monsiváis-Carrillo, A. (2020). La erosión de la democracia. Revista de Estudios Sociales, (74), 2-11. https://doi.org/10.7440/res74.2020.01

Delli Carpini, M. X. (2000). Gen.com: Youth, civic engagement, and the new information environment. Political Communication, $\quad$ 341-349. http://doi.org/10.1080/10584600050178942

Díaz Jiménez, O. F., \& Muñiz, C. (2017). Los efectos de la comunicación política en el compromiso político de los jóvenes en la elección presidencial mexicana de 2012. Revista Mexicana de Ciencias Políticas y Sociales, 62(229), 181-221. http://dx.doi.org/10.1016/So185-1918(17)300o89

Echeverría, M. (2011). ¿Apatía o desencuentro? Patrones de consumo y recepción de información política y gubernamental en jóvenes. Global Media Journal México, 8(15), 42-65.

Espinoza-Bianchini, G. (2018). Informados y confiados: El efecto del consumo de medios de comunicación tradicional y digital sobre la confianza de los chilenos en 2015. Revista Chilena de Derecho y Ciencia Política, 9(1), 208-235. https://doi.org/10.7770/rchdcp-V9N1-art1293

García Luengo, Ó. (2005). Desafectos y medios de comunicación: el estado de la cuestión de una relación difusa. Reflexión Política, 7(14), 8-24.

García Luengo, Ó. (2009). ¿Comunicando desafección? La influencia de los medios en la cultura política. Fontamara.

García-Luengo, Ó., \& Maurer, M. (2009). A virtuous circle for all? Media Exposure and political trust in Europe. CONfines, 5(9), 39-48.

Holtz-Bacha, C. (1990). Videomalaise revisited: Media exposure and political alienation in West Germany. European Journal of Communication, 5(1), 73-85. http://doi.org/10.1177/0267323190005001005

INE (2015). Informe País sobre la Calidad de la Ciudadanía en México. INE. https://portalanterior.ine.mx/archivos2/s/DECEYEC/EducacionCivica/Resumen_Ejecutivo_23nov.pdf 
Leyva, O., Muñiz, C., \& Flores, M. Á. (2016). La conformación de actitudes políticas de los jóvenes universitarios en el contexto preelectoral 2015 en Nuevo León. Revista Mexicana de Opinión Pública, (21), 51-70. http://dx.doi.org/10.1016/j.rmop.2015.10.001

Maldonado, G. (2013). Desapego político y desafección institucional en México. ¿Desafíos para la calidad de la democracia? Política y Gobierno, 20(temático), 109-138.

Marañón, F. J., Muñiz, C., \& Saldierna, A. R. (2017). La persuasión política en campañas electorales: Cuasi-Experimento sobre el efecto del uso de rutas persuasivas de los spots políticos. Daena: International Journal of Good Conscience, 12(2), 148-158.

McQuail, D. (1972). The television audience: A revised perspective. En D. McQuail (Ed.), Sociology of Mass Communications (pp. 135-165). Penguin.

Montero, J. R., Gunther, R., Torcal, M., \& Menezo, J. C. (1998). Actitudes hacia la democracia en España: legitimidad, descontento y desafección. REIS: Revista Española de Investigaciones Sociológicas, (83), 9-49.

Muñiz, C. (2019). Prácticas comunicativas y desafección política en el contexto de las campañas electorales. Análisis de su relación desde el modelo O-S-R-O-R. index.Comunicación, 9(1), 80-107. https://doi.org/10.33732/ixc/o9/o1Practi

Muñiz, C., \& Maldonado, L. (2011). Entre la movilización y el malestar mediático. Impacto de las prácticas comunicativas en las actitudes políticas de los jóvenes. Perspectivas de La Comunicación, 4(2), 32-54.

Muñiz, C., Saldierna, A. R., Marañón, F. J., \& Maldonado, L. (2016). Estudios sobre comunicación política en Latinoamérica. Fontamara.

Norris, P. (2000). A virtuous circle: Political communication in post-industrial democracies. Oxford University Press.

Norris, P. (2001). ¿Un círculo virtuoso? El impacto de las comunicaciones políticas en las democracias post-industriales. Revista Española de Ciencia Política, (4), 7-33.

Paramio, L. (1999). Cambios sociales y desconfianza política: el problema de la agregación de preferencias. Revista Española de Ciencia Política, (1), 81-95.

Pharr, S. J., \& Putnam, R. D. (2000). Disaffected democracies: What's troubling the trilateral countries? Princeton University Press.

Robinson, M. J. (1976). Public affairs television and the growth of political malaise: The case of 'The Selling of the Pentagon'. American Political Science Review, 70(02), 409-432. https://doi.org/10.2307/1959647 
Rojas, H. (2006). Comunicación, participación y democracia. Universitas Humanística, 62, 109-142.

Rosanvallon, P. (2006). La democracia inconclusa. Historia de la soberanía del pueblo en Francia. Taurus.

Saldierna Salas, A. R. (2014). El papel de la comunicación en la formación de la cultura política: análisis del efecto de los hábitos comunicativos en las actitudes políticas de los pre-ciudadanos en Nuevo León [Tesis doctoral, Universidad Autónoma de Nuevo León]. Repositorio Institucional UANL. http://eprints.uanl.mx/4071/

Saldierna, A. R., \& Muñiz, C. (2014). Impacto del consumo de medios de comunicación en las actitudes políticas, experiencias entre estudiantes de bachillerato Monterrey. En E. Pastor Seller, K. A. C. Sáenz López, \& G. Tamez González (Coords.), Gobernabilidad, ciudadanía y democracia participativa. Análisis comparado España-México (pp. 209-228). Dykinson.

Saldierna, A. R., \& Muñiz, C. (2015). Influencia de los hábitos comunicativos en la generación de participación y cinismo político entre los pre-ciudadanos. Anuario Electrónico de Estudios en Comunicación Social "Disertaciones", 8(2), 37-59. http://dx.doi.org/10.12804/disertaciones.02.2015.03

Sani, G. (1981). Cultura política. En N. Bobbio, \& N. Matteucci (Eds.), Diccionario de ciencia política. Siglo XXI.

Shah, D. V., Cho, J., Eveland, W. P., \& Kwawak, N. (2005). Information and expression in a digital age. Communication Research, 32(5), 531-565. http://dx.doi.org/10.1177/0093650205279209

Shah, D. V., Cho, J., Nah, S., Gotlieb, M. R., Hwawang, H., Lee, N.-J., \& McLeod, D. M. (2007). Campaign ads, online messaging, and participation: Extending the communication mediation model. Journal of Communication, 57(4), 676-703. http://dx.doi.org/10.1111/j.14602466.2007.00363.x

Téllez, N. M. (2017). El aprendizaje político como puente entre el visionado de los debates electorales y la participación político electoral [Tesis doctoral, Universidad Autónoma de Nuevo León]. Repositorio Institucional UANL. http://eprints.uanl.mx/13986/

Torcal, M. (2002). Institutional disaffection and democratic history in new democracies. Central European Political Science Review, 3(10), 40-77.

Torcal, M. (2006). Desafección institucional e historia democrática en las nuevas democracias. Revista SAAP, 2(3), 591-634. 
Torcal, M., \& Montero, J. r. (2006). Political disaffection in comparative perspective. En M. Torcal \& J. R. Montero (Eds.), Political disaffection in contemporary democracies: Social capital, institutions, and politics (pp. 3-20). Routledge.

Trejo, R. (2000). Los medios de comunicación en los procesos electorales de México. En. B. A. Driscoll, S. Núñez, \& J. Castro Rea (Coords.), Los procesos electorales en América del Norte en 1994 (pp. 307-314).UNAM-CISAN.

Yamamoto, M., \& Kushin, M. J. (2014). More harm than good? Online media use and political disaffection among college students in the 2008 election. Journal of Computer-Mediated Communication, 19(3), 430-445. http://doi.org/10.1111/jcc4.12046

Zukin, C. (1981). Mass communication and public opinion. En K. R. Sanders \& D. D. Nimmo (Eds.), Handbook of political communication (pp. 359-390). SAGE. 